\title{
COVID-19 and breast cancer at a Regional Breast Centre: our flexible approach during the pandemic
}

\author{
Lina Cadili ${ }^{1}$ Kristin DeGirolamo ${ }^{1} \cdot$ Elaine McKevitt $^{1,2} \cdot$ Carl J. Brown $^{3,4} \cdot$ Christopher Prabhakar $^{5,6} \cdot$ Jin-Si Pao ${ }^{1,2}$. \\ Carol Dingee $^{1,2} \cdot$ Amy Bazzarelli $^{1,2} \cdot$ Rebecca Warburton $^{1,2}$ (i)
}

Received: 15 July 2020 / Accepted: 29 October 2020 / Published online: 4 November 2020

(c) Springer Science+Business Media, LLC, part of Springer Nature 2020

\begin{abstract}
Purpose In British Columbia (BC), there have been 2790 confirmed COVID-19 cases as of June 20, 2020. The aim of this project is to capture the effect of COVID-19 on the volume of surgery and adaptations to the surgical care of patients at a breast centre in BC.

Methods All proven or suspected breast cancer cases treated with surgery between March 16, 2019 and April 30, 2019 and March 16, 2020 and April 30, 2020 through the Providence Breast Centre were included in this review. The date ranges in 2020 mark the early COVID-19 pandemic period in BC and the large shift in operating room access during this time.

Results In 2019, 99 patients underwent surgery for proven breast cancer and 30 patients for suspected breast cancer. In 2020, 162 patients underwent surgery for breast cancer and 34 for suspected breast cancer. Wait times from core biopsy to surgery and surgery to oncology consultation were improved in 2020 with a reduction of core biopsy to surgery time from 58 to 28 days for patients seen during the pandemic. There was an increased use of regional anesthesia and same day discharge compared to 2019 with increases in regional anesthesia (41\%-89\%) and same day discharge (64\%-86\%) after adaptations to the pandemic were implemented.

Conclusions Changes such as improved access to telemedicine, timing for cancer surgeries, and safer anesthetic techniques in response to the pandemic will change breast cancer surgical care beyond the pandemic era. Centralization and team-based care is the way forward.
\end{abstract}

Keywords Breast cancer $\cdot$ Health systems $\cdot$ COVID-19 $\cdot$ Breast surgery

Rebecca Warburton

RWarburton@providencehealth.bc.ca

1 Division of General Surgery, University of British Columbia, Vancouver, BC, Canada

2 Providence Breast Centre, Mount Saint Joseph Hospital, 3080 Prince Edward Street, Vancouver, BC V5T 3N4, Canada

3 Provincial Lead, Surgery, BC Cancer, $600 \mathrm{~W}$ 10th Ave, Vancouver, BC V5Z 4E6, Canada

4 General and Colorectal Surgery, St Paul's Hospital, 1081 Burrard St, Vancouver, BC V6Z 1Y6, Canada

5 Regional Anesthesia Division, Department of Anesthesiology, Providence Health Care, 1081 Burrard St, Vancouver, BC V6Z 1Y6, Canada

6 Department of Anesthesiology, Pharmacology and Therapeutics, University of British Columbia, Vancouver, BC, Canada

\begin{tabular}{ll}
\multicolumn{2}{l}{ Abbreviations } \\
BC & British Columbia \\
COVID-19 & Coronavirus Disease 2019 \\
ED & Emergency Department \\
OR & Operating Room \\
PBC & Providence Breast Centre
\end{tabular}

\section{Introduction}

Breast cancer management is multidisciplinary and requires coordination of general surgery, plastic surgery, radiology, oncology, pathology, anesthesiology, and primary care. One in 8 women are expected to develop breast cancer during their lifetime, and one in 34 are expected to die of this disease as it is the number one cancer affecting women [2]. The Coronavirus Disease 2019 (COVID-19) pandemic has placed many strains on the Canadian health care system, in particular to the flow of operating rooms [3], a resource 
needed to treat breast cancer. Mitigation strategies for treatment of breast cancer were quickly developed to help deliver appropriate care during the pandemic [4-7].

In British Columbia the operating room (OR) slow down started on March 16, 2020 and continued until May 4, 2020 allowing only urgent procedures, including oncology to proceed. Urbach and Martin describe that in Ontario, 45,000 fewer surgeries were performed in adults with non-cancerrelated disease in March and April of 2020 (a more than 90\% reduction compared to the same time period in 2019) [3]. They go on to suggest that single entry models and teambased care may be an effective way to reduce pandemic induced prolonged wait times [3].

The Providence Breast Centre (PBC) is a high-volume breast centre in Mount Saint Joseph Hospital in Vancouver, British Columbia that was established in 2009, and since this time has been providing care to women with malignant and benign breast disease $[8,9]$. The $\mathrm{PBC}$ is a multidisciplinary clinic with a centralized referral system that sees patients from across British Columbia [8, 9]. On average PBC breast surgeons perform 1100 breast surgeries annually, approximately 800 procedures for cancer and the centre sees about 9000 patient visits a year.

The pandemic required changes in delivery of breast cancer care. Meetings were held within the PBC, anesthesia, radiology, and oncology to develop and implement care pathway changes that would allow for safe and timely care. Adaptations to perioperative care included increased regional anesthesia to minimize aerosol-generating medical procedures and thus protecting patients and healthcare workers while conserving personal protective equipment. The primary outcome of this project was to assess the ability of PBC's centralized and team-based model of care to adapt to the pandemic by assessing the wait times for surgical care of breast cancer patients compared to a similar time frame in the previous year. Secondary outcomes included adaptations to perioperative care (type of anesthetic and same day surgery), the use of virtual health, and the rates of unplanned visits to the hospital after surgery.

\section{Methods}

We conducted a retrospective cohort study using our prospectively maintained breast surgery database at PBC. All patients who underwent surgery for proven breast cancer or suspected breast cancer between March 16, 2019 to April 30, 2019 and March 16, 2020 to April 30, 2020 were included. The date ranges in 2020 mark the early COVID-19 pandemic period in British Columbia and the large shift in OR and hospital access during this time. Wait time for surgical consultation and surgery was the primary outcome. Secondary outcomes included: type of surgery performed, type of anesthetic administered, and cases done as day surgery. Unplanned post-operative hospital visits were collected via a provincial electronic health record.

Wait time calculations included core biopsy proven cancer cases and excluded excisional biopsies for high-risk lesions, surgery after neoadjuvant therapy, revision surgery for margins or upstaging, reconstruction, and patient preference delays to care. The patients were divided into three groups based on their consultation date. These groups reflect changing hospital policies during the pandemic: Group 1 consultation up to March 13, Group 2 consultation between March 14 and March 27, and Group 3 consultations between March 28 and April 30. On March 15, a provincial decision was made to halt elective surgery so until this time it was OR bookings as per pre-COVID protocol. March 16-27 was only urgent cases and discussion around ways to decrease patients seen in clinics and OR to minimize use of personal protective equipment and general anesthetic risk were begun. March 30 was the start of a centralized wait list for the OR and anesthesia implementing regional approaches were started April 3 (Fig. 1) [10].

The virtual teaching consisted of teaching materials sent electronically as a PDF booklet, virtual visits with a nurse navigator and videoconferencing with physical therapy for post-surgery recovery exercises.

\section{Statistical analysis}

We performed all statistical analyses using Microsoft Excel 2020 Version 16.37. A $p$-value of less than 0.05 was considered statistically significant. When comparing 2019-2020 data, a t-test was used for continuous variables and a Chisquare or Fisher's exact test was used for categorical variables. When performing analysis of the three subgroups, ANOVA was used.

\section{Results}

Between March 16, 2019 and April 30, 2019, 99 patients underwent surgery for breast cancer, and 30 patients for suspected breast cancer (Table 1). Over this same time period in 2020, which marks the early phase of the COVID-19 pandemic, 162 patients underwent surgery for breast cancer, and 34 for suspected breast cancer. Most cancer patients underwent breast conserving surgery, with $78 \%$ in 2019 and $71 \%$ in 2020 . Reconstruction (mastectomy with immediate reconstruction by Plastic Surgery) represented $15 \%$ of cases in 2019 and $11 \%$ of cases during the 2020 pandemic. In 2019, 58 (45\%) patients required wire localization procedures for non-palpable masses and in 2020, 99 (51\%). 


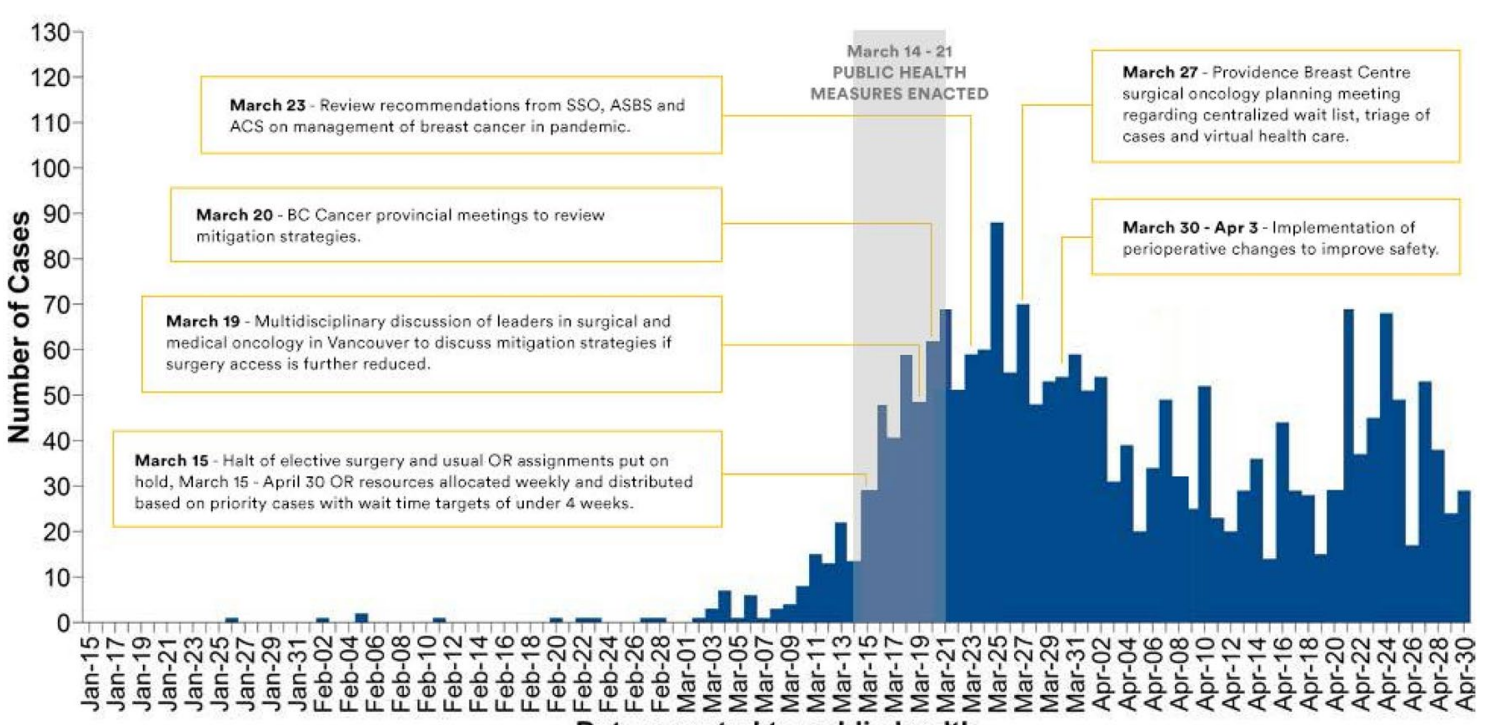

Date reported to public health

Fig. 1 Number of COVID-19 Cases in British Columbia and Healthcare System Changes

Table 1 Patient demographics and characteristics of pathology and treatment

\begin{tabular}{llllll}
\hline & $\begin{array}{l}\text { Cancer Patients } \\
2019(n=99)\end{array}$ & $\begin{array}{l}\text { Cancer Patients } \\
2020(n=162)\end{array}$ & $p$-value & $\begin{array}{l}\text { Suspected Cancer } \\
\text { Patients 2019 }(n=30)\end{array}$ & $\begin{array}{l}\text { Suspected Cancer } \\
\text { Patients 2020 }(n=34)\end{array}$ \\
\hline Sex (\% Female) & $99 / 99(100 \%)$ & $161 / 162(99 \%)$ & 0.53 & $29 / 30(97 \%)$ & $34 / 34(100 \%)$ \\
Average Age (years) & 60 & 57 & 0.24 & 48 & 46 \\
Tumor Histology & & & & 0.47 \\
DCIS & $23 / 99(23 \%)$ & $34 / 162(21 \%)$ & 0.76 & $0 / 30(0 \%)$ & $0 / 34(0 \%)$ \\
Invasive Cancer & $74 / 99(75 \%)$ & $128 / 162(79 \%)$ & 0.44 & $0 / 30(0 \%)$ & $0 / 34(0 \%)$ \\
Benign & $2 / 99$ (2\%) & $0 / 162(0 \%)$ & 0.15 & $30 / 30(100 \%)$ & $34 / 34(100 \%)$ \\
Number of Fine Wire Localizations & $45 / 99(45 \%)$ & $84 / 162(52 \%)$ & 0.37 & $13 / 30(43 \%)$ & $15 / 34(44 \%)$ \\
Type of Surgery & & & & 1 \\
Breast Conserving Surgery & $70 / 99(71 \%)$ & $127 / 162(78 \%)$ & 0.18 & $30 / 30(100 \%)$ & $34 / 34(100 \%)$ \\
Mastectomy & $29 / 99(29 \%)$ & $35 / 162(22 \%)$ & 0.18 & $0 / 30(0 \%)$ & $0 / 34(0 \%)$ \\
Reconstruction & $15 / 99(15 \%)$ & $18 / 162(11 \%)$ & 0.34 & $0 / 30(0 \%)$ & $0 / 34(0 \%)$ \\
Axillary Procedure & & & & 1 \\
SLNB & & & 1 \\
ALND & $57 / 99(58 \%)$ & $99 / 162(61 \%)$ & 0.60 & N/A & N/A \\
None & $5 / 99(5 \%)$ & $14 / 162(9 \%)$ & 0.33 & N/A & N/A \\
\hline
\end{tabular}

SLNB Sentinel Lymph Node Biopsy, ALND Axillary Lymph Node Dissection

${ }^{\text {a }}$ These two surgeries were for BRCA positive patients

Diagnostic surgery for suspected breast cancer or high-risk lesions contributed 30 cases in 2019 and 34 cases in 2020.

Table 2 illustrates that wait times were consistent between the two study periods: core biopsy to consult was 18 days for both groups $(p=0.98)$; for surgical consult to surgery it took 23 days (2019) and 27 days (2020) $(p=0.11)$. Overall wait from core biopsy to surgery was 40 days in 2019 vs 45 days in $2020(p=0.18)$. The wait time from core biopsy to BC Cancer consultation (medical oncology or radiation oncology) was 77 days in 2019 and 75 days in 2020 $(p=0.72)$. However, patients on average waited 36 days after surgery for first consultation at BC Cancer in 2019 compared to 29 days in $2020(p=0.03)$. Further analysis of the 2020 cohort revealed that 167 new surgical consults were done during this time, 60 of those were for invasive breast cancer. Ten invasive breast cancer patients seen between March 16 and April 30, 2020 were treated with neoadjuvant therapy, chemotherapy or endocrine (Table 3 ). 
Table 2 Wait times

\begin{tabular}{llll}
\hline & $\begin{array}{l}\text { Cancer Patients } \\
2019(n=69)\end{array}$ & $\begin{array}{l}\text { Cancer Patients } \\
2020(n=115)\end{array}$ & $p$-value \\
\hline Core Biopsy to Surgical Consult (average days) & 18 & 18 & 0.98 \\
Surgical Consult to Surgery (average days) & 23 & 27 & 0.11 \\
Core Biopsy to Surgery (average days) & 40 & 45 & 0.18 \\
Surgery to BC Cancer Consult $^{\text {a }}$ (average days) & 36 & 29 & 0.03 \\
Core Biopsy to BC Cancer Consult $^{\text {a }(a v e r a g e ~ d a y s) ~}$ & 77 & 75 & 0.72 \\
\hline
\end{tabular}

${ }^{\mathrm{a}} 15$ of 115 cancer patients in 2020 have not yet had a BCCA consult at the time of data collection

Table 3 Sub-group analysis of wait times in 2019 and 2020 for Cancer cases

\begin{tabular}{|c|c|c|c|c|}
\hline & 2019 & $p$-value & 2020 & $p$-value \\
\hline \multicolumn{5}{|c|}{ Core Biopsy to Surgical Consult (average days) } \\
\hline $\begin{array}{l}\text { Group } 1 \text { (consults up to Mar } \\
\text { 13) }\end{array}$ & 17 & 0.69 & 18 & 0.77 \\
\hline $\begin{array}{l}\text { Group } 2 \text { (consults Mar 14-Mar } \\
\text { 27) }\end{array}$ & 19 & & 16 & \\
\hline $\begin{array}{l}\text { Group } 3 \text { (consults Mar 28-Apr } \\
\text { 30) }\end{array}$ & 18 & & 18 & \\
\hline \multicolumn{5}{|c|}{ Surgical Consult to Surgery (average days) } \\
\hline Group 1 & 27 & 0.002 & 39 & 0.0000001 \\
\hline Group 2 & 22 & & 23 & \\
\hline Group 3 & 17 & & 11 & \\
\hline \multicolumn{5}{|c|}{ Core Biopsy to Surgery (average days) } \\
\hline Group 1 & 43 & 0.11 & 58 & 0.000003 \\
\hline Group 2 & 41 & & 39 & \\
\hline Group 3 & 35 & & 28 & \\
\hline \multicolumn{5}{|c|}{ Surgery to BC Cancer Consult (average days) } \\
\hline Group 1 & 32 & 0.46 & 32 & 0.05 \\
\hline Group 2 & 39 & & 29 & \\
\hline Group 3 & 40 & & 25 & \\
\hline \multicolumn{5}{|c|}{ Core Biopsy to BC Cancer Consult (average days) } \\
\hline Group 1 & 76 & 0.86 & 89 & 0.00001 \\
\hline Group 2 & 80 & & 66 & \\
\hline Group 3 & 76 & & 54 & \\
\hline
\end{tabular}

A subgroup analysis was performed to further examine wait times for the cancer patients in 2019 and 2020 (Table 4; Fig. 2a, b). In 2019, wait times from core biopsy to surgical consult $(p=0.69)$, core biopsy to surgery $(p=0.11)$ and surgery to BC Cancer consult $(p=0.46)$ did not change in the three time periods. The time from surgical consult to surgery $(p=0.002)$ did vary in the three time groups in 2019. This may be accounted for by OR slow down during spring break, which occurs yearly in March. In 2020, surgical consult to surgery, core biopsy to surgery and surgery to BC Cancer consult saw a decrease in most wait times as the pandemic progressed. The wait time from core biopsy to surgical consult did not change during the pandemic $(p=0.77)$.

There was increased use of regional/local anesthesia instead of general anesthesia in 2020 in order to avoid intubation during the pandemic; 93 of 162 (57\%) of cancer patients had only regional anesthetic, compared to 3 of 99 (3\%) in 2019 ( $p=0.00001)$ (Fig. 3). All the patients who had immediate reconstruction were under general anesthetic and in 2019 this included 15 patients and in 2020, 18 patients. There was a statistically significant difference in same day discharges for cancer surgery patients, $68 \%$ in 2019 vs $93 \%$ in 2020 ( $p=0.00001$ ) (Fig. 4). In 2019, two of 99 cancer patients $(2 \%)$ presented to the emergency department (ED) post-operatively and both discharged home. In 2020, 7 of 162 cancer patients (4\%) presented post-operatively to the emergency department, 5 were sent home and 2 were admitted to hospital (Table 5). No patients in 2019 had a virtual consult or received virtual teaching. In 2020, 24 patients who underwent breast surgery had a virtual consult and 22 received a virtual teaching containing information about their surgery.

Table 4 Unplanned post-operative hospital visits

\begin{tabular}{|c|c|c|c|c|c|c|}
\hline & $\begin{array}{l}\text { Cancer Patients } \\
2019(n=99)\end{array}$ & $\begin{array}{l}\text { Cancer Patients } \\
2020(n=162)\end{array}$ & $p$-value & $\begin{array}{l}\text { Suspected Cancer } \\
\text { Patients } 2019(n=30)\end{array}$ & $\begin{array}{l}\text { Suspected Cancer } \\
\text { Patients } 2020(n=34)\end{array}$ & $p$-value \\
\hline $\begin{array}{l}\text { Seen in Emergency } \\
\text { Department and } \\
\text { Discharged Home }\end{array}$ & $2 / 99(2 \%)$ & $7 / 162(4 \%)$ & 0.4987 & $1 / 30(3 \%)$ & $0 / 34(0 \%)$ & 0.4687 \\
\hline $\begin{array}{l}\text { Seen in Emergency } \\
\text { Department and } \\
\text { Admitted to Hospital }\end{array}$ & $0 / 99(0 \%)$ & $2 / 162(1 \%)$ & 0.5273 & $0 / 30(0 \%)$ & $0 / 34(0 \%)$ & 1 \\
\hline
\end{tabular}


a
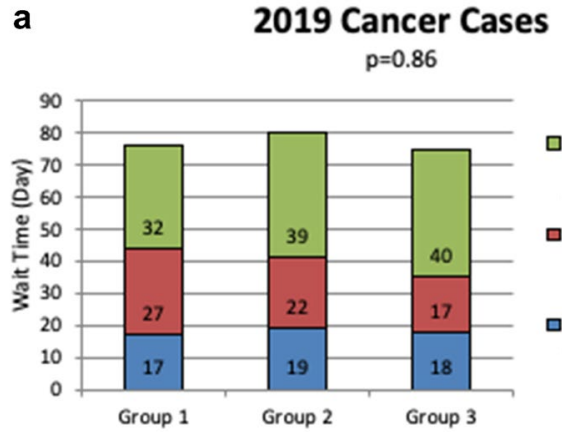

口Operation to medical/radiaiton oncology consult (BC Cancer)

口Consult to Operation

口Core Biopsy to Surgery Consult

b
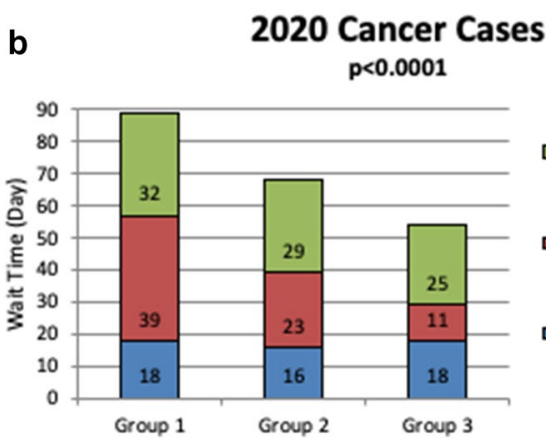

口operation to medical/radiation oncology consult (BC Cancer) $\square$ Consult to Operation

$\square$ Core Biopsy to Surgery Consult

Fig. 2 a Cancer Wait Times 2019. b Cancer Wait Times 2020

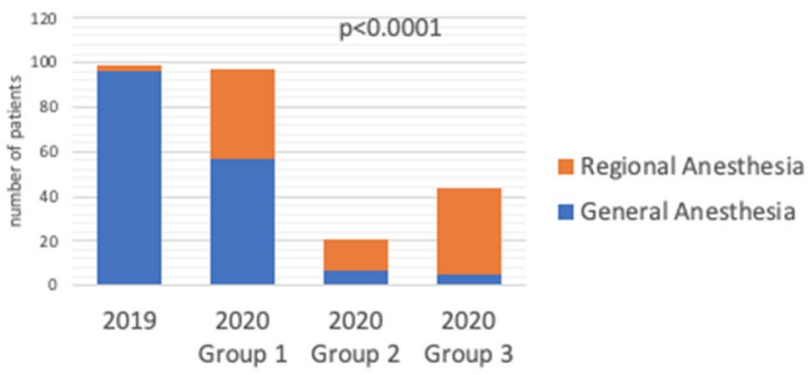

Fig. 3 Type of Anesthesia

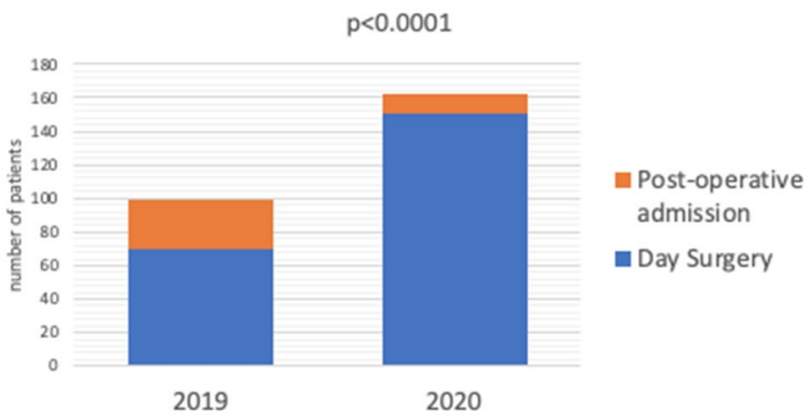

Fig. 4 Proportion of Same Day Discharge in 2019 and 2020

\section{Discussion}

COVID-19 represents a unique and challenging time in Canadian Healthcare delivery, especially for those who require a time sensitive surgery like breast cancer patients. PBC had an established centralization model for referrals and access to breast surgeons, and this foundation allowed a smooth transition during the pandemic when operative volumes increased [9]. The team-based model over individual practices at $\mathrm{PBC}$ reflects its strong interdisciplinary connections. A dynamic waitlist was created via weekly discussions with radiology, pathology, anesthesiology and OR management teams to ensure the maximum number of operative cases were being done safely. The centralized wait list and triage system ensured patients and surgeon providers were matched to reduce wait times and take advantage of excess OR time resulting from non-urgent surgery cancelations. Constant communication of the growing centralized wait list held at PBC to the health authority and BC Cancer helped build a collaborative strategy, allowing changes to be made to practice patterns in a very short amount of time. The PBC experience during the early phase of this pandemic highlights the value of team-based care and centralized services. This review is evidence that using these principles to deliver surgical service allowed quick adaptations to a rapidly changing landscape providing safe cancer care at an accelerated rate compared to the year prior.

The group managed to expand capacity and efficiently assess more breast cancer patients without increasing wait times or delaying suspected cancer patients. Initially, the pandemic called for most ORs to slow down or stop completely while issues of safety around surgery and hospital resource capacity were addressed. During this time of uncertainty mitigation strategies were developed with BC Cancer regarding management of breast cancer in the setting of reduced surgical services. Institutional decisions to proceed with only urgent elective cases (those requiring surgery within 4 weeks of consent) increased capacity for cancer patients. PBC and BC Cancer did not need to implement mitigation strategies developed only for times of reduced surgical capacity. $16.7 \%$ of new invasive cancer consults seen during the 2020 study period were treated with neoadjuvant therapy. Many societies and organizations recommended consideration of neoadjuvant therapy during the pandemic. This was not required at our institution due to safe delivery of surgical services and low impact of COVID19 in our hospital. During this time frame a maximum of 2 patients in the entire hospital were COVID-19 positive and none of the patients involved in this study contracted COVID-19 in the post-operative period.

When our data are broken down into three groups representing the changing landscape of OR availability, 
Table 5 Patients with Invasive Cancer Treated with Neoadjuvant Therapy or Surgery

\begin{tabular}{|c|c|c|c|c|c|c|}
\hline \multirow[t]{2}{*}{ New Invasive Cancer Diagnosis at PBC $(n=60)$} & \multicolumn{2}{|c|}{$\begin{array}{l}\text { Triple neg } \\
(n=8)\end{array}$} & \multicolumn{2}{|c|}{$\begin{array}{l}\text { HER2 pos } \\
(n=11)\end{array}$} & \multicolumn{2}{|c|}{$\begin{array}{l}\text { ER pos/HER2 } \\
\text { neg } \\
(n=41)\end{array}$} \\
\hline & NAT & Surgery & NAT & Surgery & NAT & Surgery \\
\hline Mar 16-Apr 30, 2020 & 3 & 5 & 3 & 8 & 4 & 37 \\
\hline
\end{tabular}

the wait time for core biopsy to surgery decreased during the pandemic. The surgeons at PBC worked closely with medical oncology to ensure neoadjuvant therapy was given promptly if needed, and if surgery first, OR time was shared and used efficiently on a weekly basis to treat the most urgent cases first. Using this model, referral to medical oncology did not differ from usual practice and mitigation strategies using systemic therapy to compensate for delayed operative management were not required. Also, the time to appointment at BC Cancer kept up with the increase in operations and even shortened time to seeing medical oncology, going from 36 to 29 days in 2020. Changes in healthcare delivery (virtual consults) and constant communication of the centralized wait list may have facilitated this shortened wait time.

PBC's strong multidisciplinary approach to cancer care allowed collaborations with the nursing team and Department of Anesthesiology to adapt the perioperative experience to keep patients safe while providing cancer treatment. The transition from mostly general anesthesia to regional anesthesia with sedation at PBC is remarkable. The use of regional anesthetic increased from 3\% in 2019 to $57 \%$ in 2020 and was $89 \%$ for patients diagnosed during the pandemic. Avoidance of general anesthetic not only limits an aerosolizing procedure to protect members of the healthcare team [11], but also helps facilitate pain control and same day discharge which increased from $67 \%$ to $93 \%$ in 2020 compared to 2019. Patients were motivated to limit hospital exposure resulting in rapid acceptance of change in admission practices and post-operative care. Of note, patients who received breast reconstruction all received general anesthesia and longer stay in hospital due to the complexity of their procedure and the involvement of the plastic surgery team. Access to breast reconstruction was altered during this time. DIEP reconstruction was halted due to length of surgery and impact on inpatient resources. Despite reduced access to immediate reconstruction during our study time, 18 patients had breast reconstruction in 2020 compared to 15 in 2019. In 2020, 15 patients had implant-based reconstruction and 3 TRAM reconstructions. All 3 TRAM patients had been booked for surgery prior to change in resources. In 2019, our team did 14 implant-based reconstructions and 1 TRAM reconstruction.

Despite the initiation of more same day discharges, we only saw 7 emergency visits with 2 resulting in surgery-related readmissions: one secondary to hematoma requiring operative intervention, discharged home the same day of the hematoma evacuation, and the second for a new diagnosis of metastatic breast cancer. The other 5 people who were seen in emergency but not admitted were broken down as follows: 3 were related to surgical complications (seroma, drain bypassing and hematoma) and 2 were seen for non-surgical or COVID-related reasons. In comparison in 2019 the service saw two emergency visits post-operatively due to seroma and wound infection and these patients did not require admission.

\section{Limitations}

COVID-19 forced Canadian Healthcare to take on new endeavors, most notably the rapid integration of telemedicine and e-health. Limited data exist as to how patients feel about telemedicine in breast cancer and a future area of study is patient satisfaction surveys during the pandemic and how the further integration of e-health care can be optimized to deliver care in Canada. We also saw more breast conserving surgeries (71\% in 2019-78\% in 2020). With unknown access to surgery, in particular autologous breast reconstructions, many patients elected for breast conserving surgery as capacity for this surgery was more reliable. Some patients were only examined on the day of surgery due to limited capacity of in-clinic visits and patient comfort with health care exposures during the pandemic. The downstream effect of positive margin rates and repeat surgery will need to be studied. Increasing the number of patients sent home the same day also increased ED visits to $3.6 \%$ from $2.3 \%$ in the post-operative period. Due to limitations in provincial level electronic health records, patients visiting EDs in other health authorities may not have been captured if this visit was not communicated to the surgeon. This is a limitation that requires a system change in order to capture provincial data which are currently not possible in BC.

\section{Future directions}

Many changes were undertaken during COVID-19 and our paper attempts to address these. BC in particular is very geographically large and as we centralize services, using 
more telemedicine may be cost saving if it allows patients in rural communities to maximize care in their own towns. Sabesan et al. nicely describe how virtual care led to efficient and convenient cancer care for patients in rural settings [12] and can likely be applied in BC. Patient satisfaction surveys are a necessary next step in optimizing how best to deliver both online and in-person healthcare. Future study on the potential need for second operations due to the high uptake of breast conserving surgery during the pandemic is needed and will be captured in a follow up analysis. Finally, we note the long wait times in 2020 for patients seen before the pandemic and we need to continue to optimize wait times for cancer care as health care delivery normalizes.

\section{Conclusion}

COVID-19 and its impact on healthcare, especially cancer care, will be historic for Canada. To increase breast cancer care during the pandemic, adaptations at our centre were made in surgery, oncology, radiology, pathology and anesthesia. Changes such as improved access to telemedicine, improved timing for cancer surgeries and safer anesthetic techniques will change breast cancer surgical management beyond the pandemic era. Centralization and team-based care is the way forward; it allows for adaptation in a changing environment such as a pandemic. Our model shows that this can be productive, efficient and provide safe care during a pandemic and can guide how to change care for our future patients.

Acknowledgements The authors would like to acknowledge the input of the physicians and allied health staff in the Departments of Anesthesia, Oncology, Pathology, Surgery and Radiology in our hospital and surrounding area that contributed to the development of our clinical pathways. We would also like to acknowledge Mabel Zhang who helped with data collection and manuscript preparation and Jillian Acquino for helping with figure graphic design.

Author contributions All authors contributed to the study conception and design. LC, KD, EM and RW performed material preparation, data collection and analysis. LC and KD has written the first draft of the manuscript and all authors commented on previous versions of the manuscript. All authors read and approved the final manuscript.

\section{Compliance with ethical standards}

Conflict of interest The authors of this manuscript have no conflicts of interest to declare for this publication. This study received no funding.

Ethics approval This study was approved by the Research Ethics Board by the University of British Columbia (H20-01415) and the Providence Health Care Research Institute. All procedures performed in the studies involving human participants were in accordance with the ethical standards of the institutional and/or national research committee and with the 1964 Helsinki declaration and its later amendments or comparable ethical standards.

Informed consent Informed consent was obtained as appropriate from all participants in the study as per the Research Ethics Board Standards.

\section{References}

1. Government of Canada. Epidemiological summary of COVID19 cases in Canada (2020) https://health-infobase.canada.ca/covid -19/epidemiological-summary-covid-19-cases.html. Accessed 20 June 2020.

2. BC Cancer Provincial Health Services Authority. Statistics by Cancer Type - Breast (2020) Cancer Surveillance and Outcomes, Population Oncology. https://www.bccancer.bc.ca/statistics-andreports-site/Documents/Cancer_Type_Brain_2017_20200327. pdf. Accessed 1 June 2020.

3. Urbach DR, Martin D (2020) Confronting the COVID-19 surgery crisis: time for transformational change. CMAJ 192:E585-E586

4. COVID-19 Pandemic Breast Cancer Consortium's Considerations for Re-entry (2020) The American Society of Breast Surgeons. https://www.breastsurgeons.org/docs/covid19/reentry.pdf?v1. Accessed 1 June 2020.

5. COVID 19: Elective Case Triage Guidelines for Surgical Care. Breast Cancer Surgery (2020) American College of Surgeons. https://www.facs.org/covid-19/clinical-guidance/elective-case. Accessed 1 June 2020.

6. ESMO Management and Treatment Adapted Recommendations in the COVID-19 Era: Breast Cancer (2020) European Society for Medical Oncology. https://www.esmo.org/guidelines/cancer-patie nt-management-during-the-covid-19-pandemic/breast-cancer-inthe-covid-19-era. Accessed 1 June 2020.

7. Provincial Cancer Clinical Management Guidelines in Pandemic Situation (COVID-19) (2020) British Columbia Cancer Agency. https://www.bccancer.bc.ca/health-professionals-site/Docum ents/provincial_cancer_clinical_management_guidelines_pande mic_situation_covid19_april20_2020.pdf. Accessed 1 June 2020.

8. Providence Breast Centre at Mount Saint Joseph Hospital. https ://www.msjbreastclinic.com/

9. Cha J, McKevitt E, Pao J, Dingee C, Bazzarelli A, Warburton R (2020) Access to surgery following centralization of breast cancer surgical consultations. Am J Surg 219:831-835

10. BC Centre for Disease Control. British Columbia COVID-19 Daily Situation Report, May 1, 2020. bccdc.ca/Health-Info-Site/ Documents/BC_Surveillance_Summary_May_1_2020_final.pdf. Accessed 1 June 2020.

11. COVID-19 Recommendations during Airway Manipulation. Canadian Anesthesiologists' Society (2020) https://www.cas. ca/en/practice-resources/news/cas-articles/2020/covid-19-recom mendations-during-airway-manipulation. Accessed 1 June 2020.

12. Sabesan S, Larkins S, Evans R et al (2012) Telemedicine for rural cancer care in North Queensland: bringing cancer care home. Aust J Rural Health 20:259-264

Publisher's Note Springer Nature remains neutral with regard to jurisdictional claims in published maps and institutional affiliations. 